\title{
Interferometric Imaging of Nearby Low-Mass Spectroscopic Binaries. I. KPNO Observations
}

\author{
A. DUQUENNOY ${ }^{1}$, J. CHRISTOU ${ }^{2}$, J.-M. MARIOTTI ${ }^{3}$, \\ M. MAYOR ${ }^{1}$, C. MORBEY ${ }^{4}$, C. PERRIER ${ }^{4}$, R. PROBST ${ }^{6}$, \\ E. TESSIER ${ }^{5}, \&$ S. RIDGWAY 6 \\ 1 Geneva Observatory, CH-1290 Sauverny, Switzerland \\ 2 National Optical Astronomy Observatories, Tucson, AZ 85726, USA \\ 3 Paris-Meudon Observatory, F-92195 Meudon Cedex, France \\ 4 Dominion Astrophysical Observatory, Victoria, B.C., Canada \\ 5 Grenoble Observatory, F-38041 Grenoble, France \\ 6 Kitt Peak National Observatory, Tucson, AZ 85726, USA
}

ABSTRACT: We present a preliminary report on radial-velocity and infrared interferometric observations, with emphasis on the newly resolved nearby sources Gl 609.2 and $\mathrm{Gl} \mathrm{804.} \mathrm{We} \mathrm{briefly} \mathrm{discuss} \mathrm{their} \mathrm{low-mass} \mathrm{companions,} \mathrm{their} \mathrm{luminosities,}$ and their individual masses inferred from the combined solution of their spectroscopic and visual orbits.

\section{INTRODUCTION}

The search for companions to nearby low-mass stars is of high astrophysical importance. Among others, it adresses three questions:

1. the binary rate for the red end of the Hertzprung-Russell diagram which forms the major population of the solar neighbourhood;

2. the derivation of the mass-luminosity relationship for masses between 0.08 and $0.5 \mathcal{M}_{\odot}$ and of the exact cut-off between stellar and substellar mass distributions; and

3. the existence and characteristics of brown dwarfs, for which direct observational evidences remain controversial.

Since 1977 , an on-going high-precision $\left(0.3\right.$ to $\left.0.8 \mathrm{~km} \mathrm{~s}^{-1}\right)$ radial-velocity (RV) survey of F-to-M dwarf stars contained in the Gliese (1969) catalogue, has been devoted to the study of stellar duplicity (e.g., the results on nearby G-dwarfs by Duquennoy \& Mayor 1991, and Mayor et al., this Colloquium).

As a by-product of this 15-yr spectroscopic survey, new long-period binaries have been detected or suspected. Some of them contain probable very low-mass companions which are candidates for direct detection by infrared interferometry. For that purpose, three lists for a total of 70 sources were selected to be imaged at different observatories in order to improve the knowledge of the mass/luminosity relationship for very low masses: ESO-La Silla since 1989 (see poster II, Perrier et al., this Colloquium), Calar Alto since 1990 (with Ch. Leinert, data not presented yet), KPNO in 1990-1991 (this paper). 

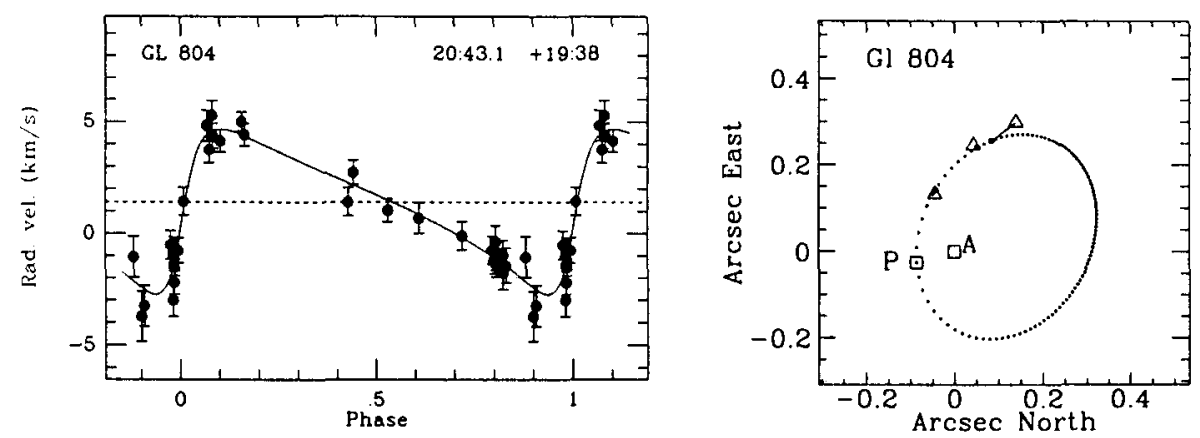

FIGURE 1. Velocity curve and orbital motion for the star Gl 804.

\section{OBSERVATIONS}

We have imaged 14 of these selected sources with the IR speckle camera of the Mayall 4-m telescope at Kitt Peak National Observatory. The diffraction limit for such an aperture and at a wavelength of $2.2 \mu \mathrm{m}$ is 0 ".1. The detector used was the Infrared Speckle Imager of the National Optical Astronomy Observatories, a $58 \times 62$ pixels InSb detector array of excellent cosmetic quality, and with basically no flat-fielding necessary.

The interferometric observations were made in June 1990 and February 1991. Three sources were resolved, of which Gl 609.2 and Gl 804 for the first time. The data treatment made use of classical speckle methods. The orbital solution for Gl 804 is shown in Figure 1: in the right panel, dots correspond to the calculated apparent position at each 0.01 phase, open triangles to the KPNO observations, $\mathrm{A}$ and $\mathrm{P}$ to the positions of the primary and of periastron.

\section{PRELIMINARY RESULTS}

Preliminary results of this study are given in Table 1. With only three observed positions, the individual masses are still poorly constrained. Figure 2 presents four of our target stars (triangles) in the HR diagram, together with the known low-mass binaries, as reprinted from Henry \& McCarthy (1990). In this figure, minimum masses are derived from the spectroscopic mass functions, upper masses are provisional, hatched areas denote the range of luminosities derived from speckle observations. The short-dashed paths are the loci of dwarf stars at ages $10^{8}$ and $5 \times 10^{9} \mathrm{yr}$, from D'Antona \& Mazitelli (1985). Note that the components of the binary Gl 473 probably have stellar masses (Henry et al. 1992, Perrier et al. 1991), so that presently no definite brown dwarf has been imaged yet. The detailed version of this study is planned in a further paper. 
TABLE 1. An overview of the characteristics of the present survey

\begin{tabular}{|lc|}
\hline Number of sources & 14 \\
Spectral types of primaries & dG3 - dM5 \\
\hline Background: CORAVEL orbits & \\
- well defined / ill defined & $8 / 6$ \\
- range of orbital periods & $1-14$ yrs \\
- expected angular separations & $0^{\prime \prime} .04-0^{\prime \prime} .60$ \\
- minimum secondary masses & $0.03-0.37 \mathcal{M}_{\odot}$ \\
\hline KPNO preliminary results: & $2 / 2$ \\
- resolved / marginally resolved sources & $3 / 7$ \\
\hline
\end{tabular}

\begin{tabular}{|ccccccc|}
\hline Results for resolved binaries: & $\mathrm{P}(\mathrm{yrs})$ & $\mathrm{a}^{\prime \prime}$ & $M_{K_{1},}$ & $M_{K, 2}$ & $\mathcal{M}_{1} / \mathcal{M}_{\odot}$ & $\mathcal{M}_{2} / \mathcal{M}_{\odot}$ \\
\hline Gl 609.2 & 12.2 & 0.26 & 4.1 & 6.1 & 0.8 & 0.3 \\
Gl 804 & 12.3 & 0.28 & 5.7 & 7.9 & 0.4 & 0.2 \\
\hline
\end{tabular}

\section{REFERENCES}

D'Antona F. \& Mazitelli I. 1985, $A p J, 296,502$

Duquennoy A. \& Mayor M. 1991, A\&A, 248, 485

Gliese W. 1969, Veröff. Astron. Reichen Inst. Heidelberg, No. 22

Henry T.J. \& McCarthy D.W. 1990, $A p J, 350,334$

Henry T.J., Johnson, D.S., McCarthy D.W., \& Kirkpatrick, J.D. 1992, A\&A, 254, 116

Morbey C.L. 1975, PASP, 87, 689

Perrier C., Mariotti J.-M., Bonneau D., \& Duquennoy A. 1991, ESO Conf. on High Resolution Imaging by Interferometry II, J. Beckers, Garching

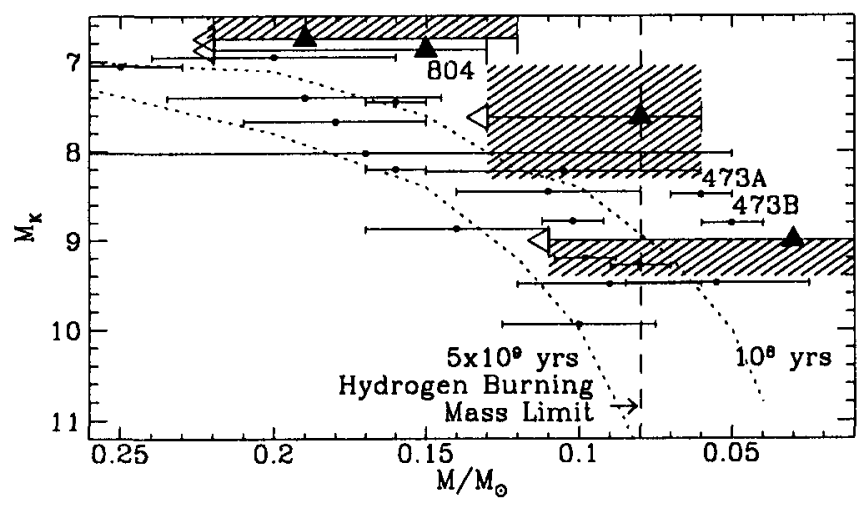

FIGURE 2. Four of our target stars in the HR diagram (see text) 